\title{
Curved Duct Noise Prediction Using the Fast Scattering Code
}

\author{
M. H. Dunn* \\ Ana F. Tinetti ${ }^{*}$ \\ Hampton, VA 23666 \\ F. Farassat ${ }^{\dagger}$ \\ NASA Langley Research Center, Hampton, VA 23681
}

\begin{abstract}
Results of a study to validate the Fast Scattering Code (FSC) as a duct noise predictor, including the effects of curvature, finite impedance on the walls, and uniform background flow, are presented in this paper. Infinite duct theory was used to generate the modal content of the sound propagating within the duct. Liner effects were incorporated via a sound absorbing boundary condition on the scattering surfaces. Simulations for a rectangular duct of constant cross-sectional area have been compared to analytical solutions and experimental data. Comparisons with analytical results indicate that the code can properly calculate a given dominant mode for hardwall surfaces. Simulated acoustic behavior in the presence of lined walls (using hardwall duct modes as incident sound) is consistent with expected trends. Duct curvature was found to enhance weaker modes and reduce pressure amplitude. Agreement between simulated and experimental results for a straight duct with hard walls (no flow) was excellent.
\end{abstract}

\section{Nomenclature}

A

$x, y, z$

$\delta$

$\rho$

$\sigma$

$\omega$

$\xi, \eta, \zeta$

Subscripts

0

$m$

$n$

$=$ Wave amplitude; also, complex admittance, $A=1 / Z$

$=$ Wave amplitude

$=$ Waveguide dimension

$=$ Mach number

$=$ Specific acoustic resistance

$=$ Region inside rectangular waveguide

$=$ Specific acoustic reactance

$=$ Specific acoustic impedance, $Z=R+X i$

$=$ Speed of sound inside duct

$=$ Excitation frequency, $\mathrm{Hz}$

$=$ Imaginary unit, $\sqrt{-1}$

$=$ Monopole index

$=$ Wave number

$=$ Pressure

$=$ Time

$=$ Cartesian coordinates

$=$ Kronecker delta

$=$ Air density inside duct

$=\operatorname{sgn}\left(z-z_{0}^{j}\right)$, a notational convenience

$=$ Excitation frequency, $2 \pi f$

$=$ General coordinates

$=$ Location of monopole source

$=$ Order of mode in $x$ direction

$=$ Order of mode in $y$ direction

*. Consultant, Senior Member AIAA

$\dagger$. Senior theoretical aeroacoustician, Fellow AIAA 


$\begin{array}{lll}x, y, z & = & \text { Cartesian coordinate directions } \\ \alpha & = & \text { Monopole strength } \\ \infty & \text { Freestream quantity } \\ \text { Superscripts } & \\ , \quad & \text { Acoustic variable } \\ \rightarrow \quad & \text { Vector }\end{array}$

\section{I - Introduction}

An accurate determination of sound as it propagates within waveguides of arbitrary geometry is of paramount importance to the development of noise reduction concepts for turbofan bypass ducts. Of interest are the effects associated with changes in duct shape, wall acoustic treatment, and the presence of components such as splitters and outlet guide vanes.

An experimental study of noise abatement concepts for bypass ducts is costly and time consuming. Taking advantage of a recently developed computational method for predicting the scattered acoustic field generated by the interaction of a known source and an arbitrary collection of surfaces ${ }^{1}$, a multitude of configurations can be explored in a timely manner, giving the engineer the ability to select only the best candidates for experimental evaluation. The prediction methodology, which uses the equivalent source method (ESM) to solve an exterior Helmholtz equation boundary value problem (BVP), has been implemented into a computer program called the Fast Scattering Code (FSC). The code uses pre-defined component geometries and operating conditions to simulate the time-harmonic scattered acoustic pressure and velocity fields at user specified locations. Details on the methodology and program usage are given in references 1 through 3.

The main objective of this investigation is to validate the FSC as a duct noise predictor, including the effects of duct curvature, acoustic treatment, and background flow. To do so, simulations for a duct with a constant, rectangular cross-section will be compared to analytical results and experimental data obtained with the NASA Langley Research Center (LaRC) Curved Duct Test Rig (CDTR) ${ }^{4}$. For the present study, the coordinate system follows FSC convention, that is, $x$ increases "up", $y$ increases spanwise, and $z$ increases upstream. The dimensions of the duct are similar to those of the CDTR $\left(L_{x}, L_{y}, L_{z}=0.381 \mathrm{~m}, 0.1524 \mathrm{~m}, 3.0 \mathrm{~m}\right)$. The length of the test section (where the liner specimens are placed) is $0.8128 \mathrm{~m}$. For simplicity, the origin lies on the duct axis, at the center of the test section. Harmonic waves of the form $e^{i \omega t}$ are assumed.

\section{II - Validation with Analytical Results}

\section{A. Infinite Duct Theory}

In general, the simulation of a scattered acoustic field using the FSC requires discretization of the surface(s) according to excitation frequency and surface area, and the generation of a suitable sound source. For constant area duct applications, the former requirement is easily performed using grid generation software; the latter can be simulated using combinations of simple monopole sources to produce duct modes of arbitrary order, amplitude, and phase. Consider a waveguide of infinite length, centered at the origin:

$$
(x, y, z) \in S \equiv\left[-\frac{L_{x}}{2}, \frac{L_{x}}{2}\right] \times\left[-\frac{L_{y}}{2}, \frac{L_{y}}{2}\right] \times(-\infty, \infty)
$$

For one monopole at $\left(x_{0}, y_{0}, z_{0}\right) \in S$, the acoustic pressure is given as ${ }^{5}$ :

$$
p^{\prime}(x, y, z)=\sum_{m=0}^{\infty} \sum_{n=0}^{\infty} A_{m n}\left(x_{0}, y_{0}\right) A_{m n}(x, y) e^{i k_{z}^{m n}\left|z-z_{0}\right|}
$$

Where

$$
A_{m n}(\xi, \eta)=\frac{2 \cos \frac{m \pi}{L_{x}}\left(\xi-\frac{L_{x}}{2}\right) \cos \frac{n \pi}{L_{y}}\left(\eta-\frac{L_{y}}{2}\right)}{\sqrt{i k_{z}^{m n} L_{x} L_{y}\left(1+\delta_{m 0}\right)\left(1+\delta_{n 0}\right)}}
$$




$$
k_{z}^{m n}=\sqrt{k^{2}-\left(\frac{m \pi}{L_{x}}\right)^{2}-\left(\frac{n \pi}{L_{y}}\right)^{2}}
$$

By judicious choice of $\left(x_{0}, y_{0}\right)$, certain modes can be silenced by making $A_{m n}\left(x_{0}, y_{0}\right)=0$ ( $m$ and $n$ not both zero). For example, to silence the $n$-th mode, $y_{0}$ can be chosen so that

$$
\frac{n \pi}{L_{y}}\left(y_{0}-\frac{L_{y}}{2}\right)= \pm \frac{\pi}{2} \Rightarrow y_{0}= \pm \frac{L_{y}}{2}\left(1-\frac{1}{n}\right), n \geq 1
$$

For $J$ monopoles with strengths $\alpha_{j}$, the acoustic pressure can be expressed as:

$$
\begin{gathered}
p^{\prime}(x, y, z)=\sum_{m=0}^{\infty} \sum_{n=0}^{\infty} \sum_{j=1}^{J} \alpha_{j} B_{m n}^{\sigma_{j}}\left(\vec{x}_{0}\right) B_{m n}^{-\sigma_{j}}(\vec{x}) \\
B_{m n}^{\sigma_{j}}(\vec{\xi})=A_{m n}(\xi, \eta) e^{i \sigma_{j} k_{z}^{m n} \zeta} \\
\sigma_{j}=\operatorname{sgn}\left(z-z_{0}^{j}\right)
\end{gathered}
$$

To silence multiple modes in regions of space for which $\mathrm{z}<\min z_{0}^{j}$,

$$
\left.p^{\prime}(x, y, z)=\sum_{m=0}^{\infty} \sum_{n=0}^{\infty}\left[\sum_{j=1}^{J} \alpha_{j} B_{m n}^{-} \stackrel{\vec{x}}{x_{0}}\right)\right] B_{m n}^{+}(\vec{x})
$$

For example, two sources can be used to silence the $(0,0)$ mode:

$$
\left.\left.\alpha_{1} B_{00}^{-} \stackrel{{ }_{1}}{x_{0}}\right)+\alpha_{2} B_{00}^{-} \stackrel{\vec{x}_{0}}{x_{0}}\right)=0 \Rightarrow \alpha_{2}=-\alpha_{1} \frac{B_{00}^{-}\left(\vec{x}_{0}\right)}{B_{00}^{-}\left(x_{0}\right)}
$$

Various dominant duct modes, excitation frequencies, background flow speeds, and wall impedances were used in the present investigation. The modes, frequencies, and flow settings were chosen from a subset of experimental data obtained using the CDTR with a straight test section; the impedances were educed from measurements taken at the NASA LaRC Grazing Incidence Tube (GIT) facility ${ }^{6}$ for a single degree of freedom, locally reacting liner built in-house. The liner, denoted as $\mathrm{L} 1^{7}$, has a honeycomb core 1.5 inches deep covered with an $8.7 \%$ open perforate. The parameter values are summarized in Table 1.

Table 1. - Parameters used in validation study.

\begin{tabular}{|l|c|c|c|c|c|c|}
\hline Excitation frequency & \multicolumn{2}{|c|}{$1500 \mathrm{~Hz}$} & \multicolumn{2}{c|}{$2000 \mathrm{~Hz}$} \\
\hline Duct mode & \multicolumn{2}{|c|}{$(0,0)$} & \multicolumn{2}{|c|}{$(2,0)$} & \multicolumn{2}{c|}{$(2,1)$} \\
\hline $\begin{array}{l}\text { Normalized Liner } \\
\text { impedance, } \mathrm{M}_{\infty}=0.0\end{array}$ & $\infty+0.0 \mathrm{i}$ & $0.23-0.14 \mathrm{i}$ & $\infty+0.0 \mathrm{i}$ & $0.23-0.14 \mathrm{i}$ & $\infty+0.0 \mathrm{i}$ & $0.12+0.35 \mathrm{i}$ \\
\hline $\begin{array}{l}\text { Normalized Liner } \\
\text { impedance, } \mathrm{M}_{\infty}=0.275\end{array}$ & $\infty+0.0 \mathrm{i}$ & $0.76-0.43 \mathrm{i}$ & $\infty+0.0 \mathrm{i}$ & $0.76-0.43 \mathrm{i}$ & $\infty+0.0 \mathrm{i}$ & $0.73+0.13 \mathrm{i}$ \\
\hline
\end{tabular}

At an excitation frequency of $1500 \mathrm{~Hz}$, several modes are cut on: $(0,0),(1,0),(2,0),(0,1),(1,1),(3,0)$, and $(2$, $1)$. The $n=1$ modes, $(0,1),(1,1)$ and $(2,1)$, are silenced by placing one source at $y_{0}=0.0$; the odd $m$ modes, $(1,0)$ and $(3,0)$, are silenced by placing the source at $x_{0}=0.0$. The remaining modes, $(0,0)$ and $(2,0)$, are alternately silenced using equation (10). At an excitation frequency of $2000 \mathrm{~Hz}$, two additional modes are cut on, (3, 1) and (4, $0)$. Four monopoles can be used to isolate the $(2,1)$ mode. Placing the sources at $y_{0} \neq 0$ ensures generation of the $n=$ 1 modes; positioning one pair of sources at $x_{0}= \pm 3 L_{x} / 8$ silences the $(1,0),(3,0),(1,1)$, and $(4,0)$ modes. The addition of a second pair of sources at the same $\left(x_{0}, y_{0}\right)$ but different $z_{0}$ silences the $(2,0)$ mode. Modal decomposition indicates that, of the remaining modes, $(0,0),(0,1)$, and $(2,1)$, the amplitude of the $(2,1)$ mode is approximately 5 times larger than that of the next strongest one. 


\section{B. Straight Duct}

Mode propagation patterns at the walls and bisecting plane of a hard-wall, straight duct for excitation frequencies of $1500 \mathrm{~Hz}$ and $2000 \mathrm{~Hz}$, in the absence of flow, are shown in Figure 1and 2 for dominant $(0,0),(2,0)$, and $(2,1)$ modes. The axial placement of the acoustic sources, $z_{0}^{1}$ and $z_{0}^{2}$, corresponds to the locations of the two driver planes in the CDTR rig. The test section inlet (in) and outlet (out), indicated in the figures, are at $z=0.4064 \mathrm{~m}$ and -0.4064 $\mathrm{m}$, respectively. Although reflections from the duct openings may be present, note from Figure 1a that the FSC is able to simulate the proper behavior of a dominant, aft travelling plane wave. Higher order modes are correctly simulated as well. Figure $1 \mathrm{~b}$ indicates that a clearly dominant $(2,0)$ mode is established immediately aft of the monopole source combination. The presence of several modes can be observed in Figure 2. As indicated by infinite duct theory, the (2, 1) mode appears dominant on the walls of the duct, and a plane wave of amplitude approximately $1 / 5$ that of the $(2,1)$ mode dominates the propagation behavior at the bisecting plane, which coincides with a node of the $(2,1)$ mode.
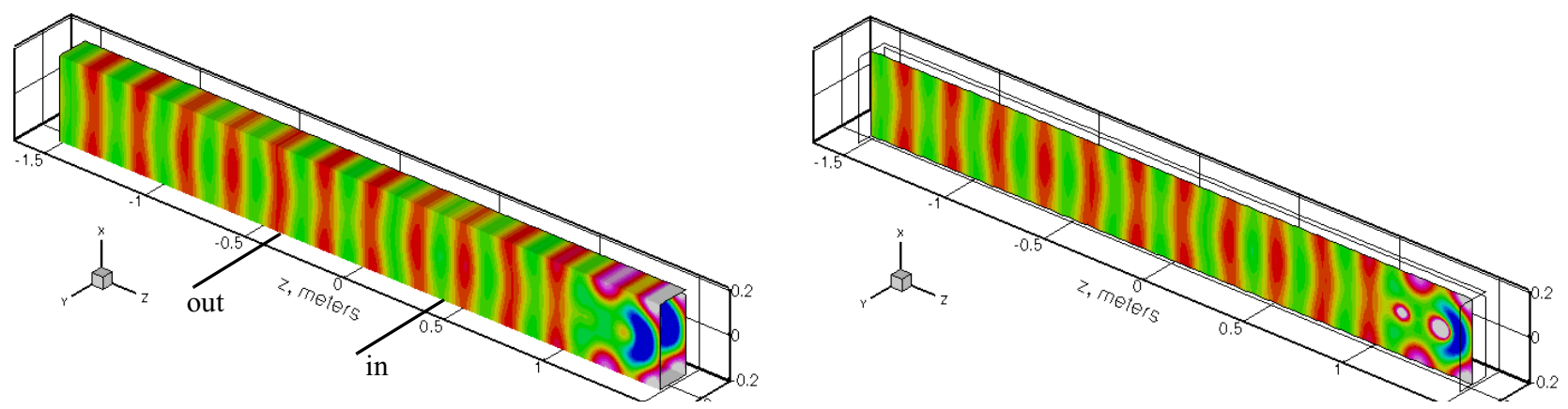

(a) Dominant $(0,0)$ mode

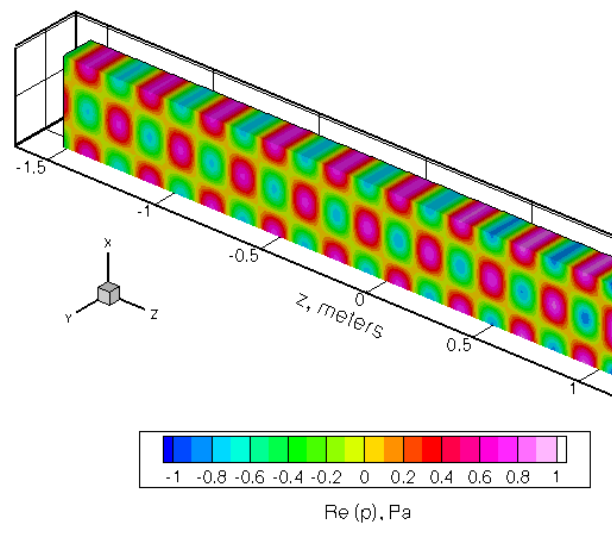

(b) Dominant $(2,0)$ mode

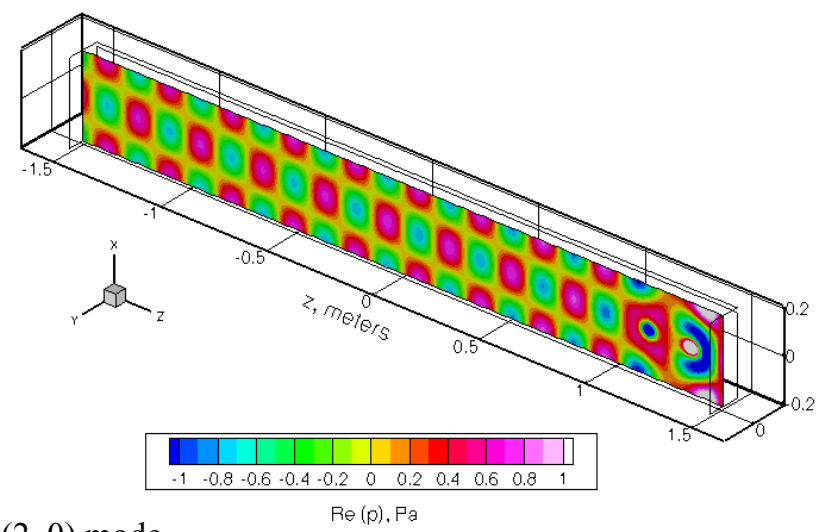

Figure 1. - Mode propagation within a hardwall, straight duct; $f=1500 \mathrm{~Hz}, M_{\infty}=0.0$.
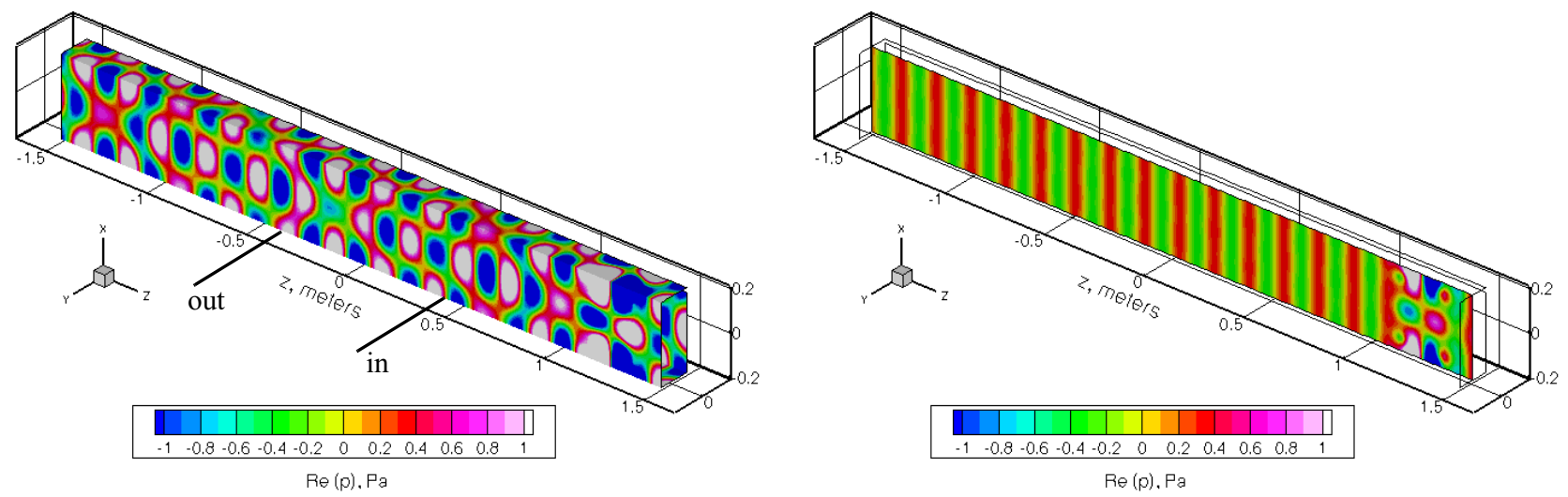

Figure 2. - Mode propagation within a hardwall, straight duct; $f=2000 \mathrm{~Hz}, M_{\infty}=0.0$, dominant $(2,1)$ mode. 
The experimental rig is equipped with a flow system capable of delivering air at speeds typical of aft fan bypass duct flows. The effects of background flow $\left(M_{\infty}=0.275\right)$ on noise propagation inside the straight duct are depicted in Figures 3 and 4. In the laboratory-fixed frame of reference, air flows from right to left (i.e., in the -z coordinate direction). For the same source locations as above, note from Figures $3 a, 3 b$, and 4 that the main effects of flow appear to be 1) a reduction in the pressure amplitudes inside the duct, 2) a decrease in wave length upstream of the sources, and 3 ) an increase in wavelength downstream of the sources. A point monopole source at rest emits an omnidirectional sound field. If the source is in motion, the radiated sound field is distorted so that pressure perturbations forward of the source are more intense ${ }^{8}$ (with reduced wavelengths) than those aft of it (with increased wavelengths), as observed in the predictions.
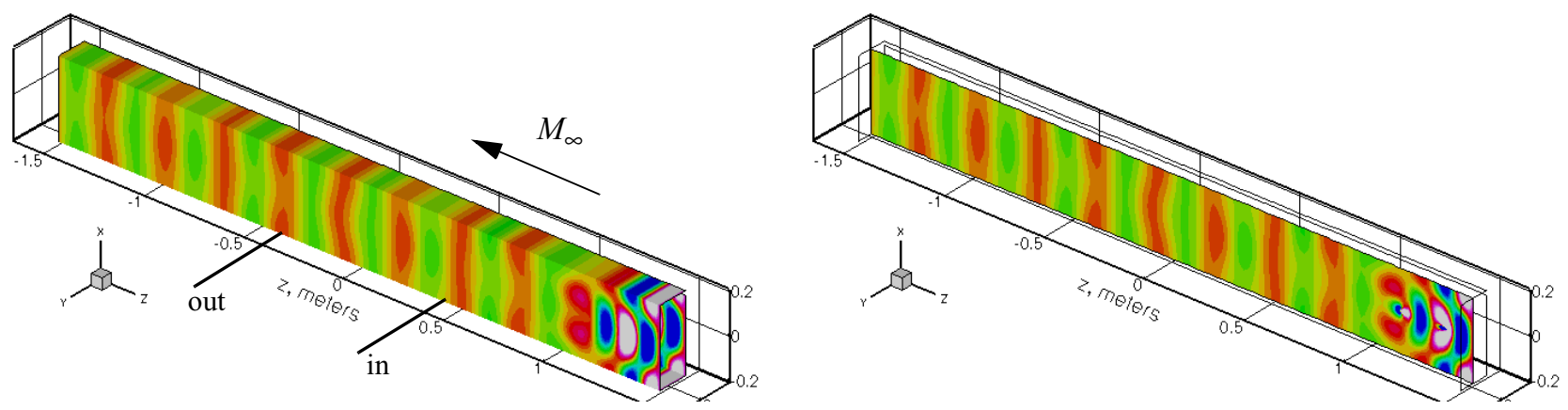

(a) Dominant $(0,0)$ mode
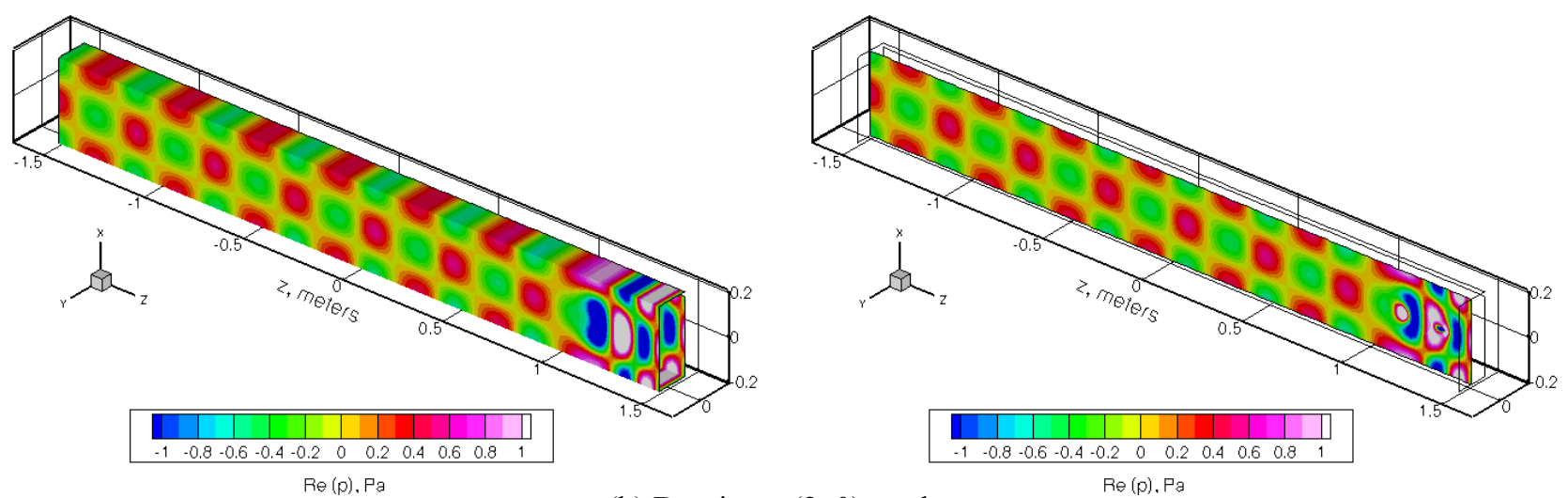

(b) Dominant $(2,0)$ mode

Figure 3. - Mode propagation within a straight duct with hard walls; $f=1500 \mathrm{~Hz}$, $M_{\infty}=0.275$ (air flows in the $-\mathrm{z}$ direction).
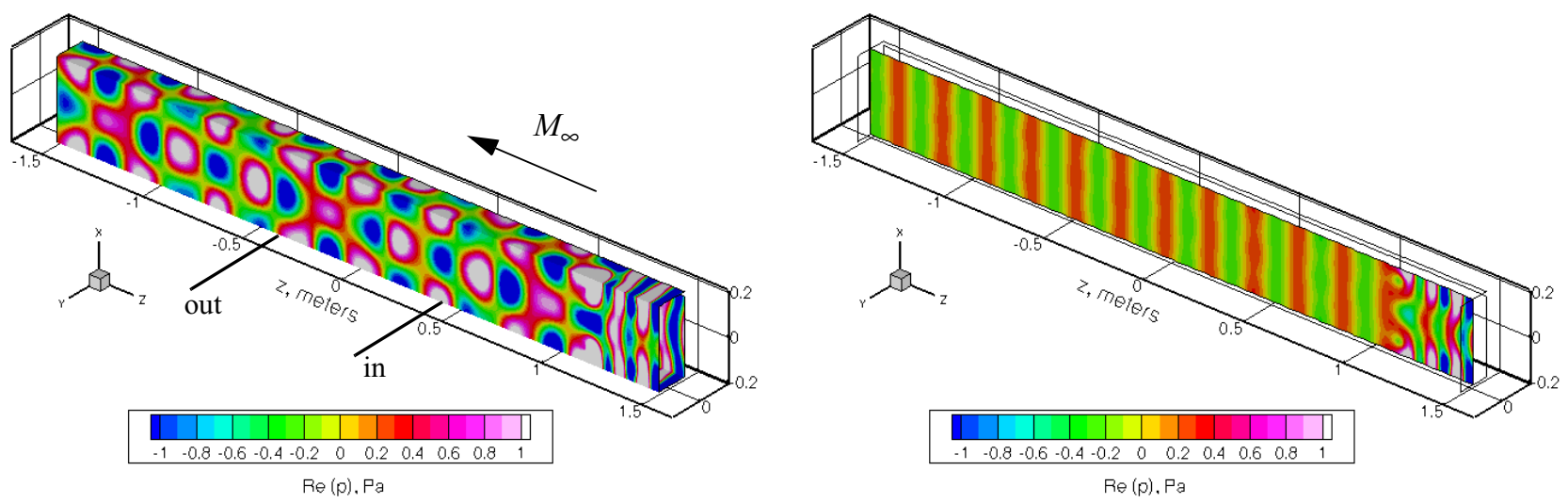

Figure 4. - Mode propagation within a straight duct with hard walls; $f=2000 \mathrm{~Hz}$, $M_{\infty}=0.275$ (air flows in the -z direction), dominant $(2,1)$ mode. 
The sound absorbing boundary condition imposed on the scattering surfaces ${ }^{3}$ requires the input of dimensional admittance, $A$. To simulate hard walls, $A=0.0+0.0 i$. To simulate acoustically treated walls, the normalized impedances of Table 1 are converted to admittances using environmental variables (air density, $\rho$, and sound speed, c, inside the duct) measured during CDTR tests. For the present exercise, the incident acoustic field is in the form of hardwall duct modes.

The effects of liner treatment for the entire side walls of the test section (the top and bottom walls remain hard) are shown in Figures 5 through 7 for $M_{\infty}=0.0$. For a given frequency, the peak attenuation of a liner is proportional to the length and surface area of the treatment ${ }^{9,10}$. It has also been determined ${ }^{9}$ that the initial portion of the treatment, in the vicinity of the test section entrance, is generally more effective because the higher order modes are attenuated more rapidly than the remaining - predominantly plane-wave - propagating modes. Note from Figure 5 that, for a dominant $(0,0)$ mode, the effect of the liner increases gradually from the test section inlet plane $(z=0.4064 \mathrm{~m})$, peaks at approximately $\mathrm{z}=0.0 \mathrm{~m}$, and remains more or less constant after that. The sound attenuation through the treated test section appears to be on the order of $30 \mathrm{~dB}$. Similar behavior is observed for a dominant $(2,0)$ mode (Figure 6), with a slightly larger peak attenuation $(35 \mathrm{~dB})$. When the $(2,1)$ mode is dominant in the duct, the liner seems to abate all modes except for the plane wave, as clearly seen in the acoustic pressure contours of Figure 7 . This results in a relatively modest sound reduction through the test section, approximately $5 \mathrm{~dB}$.
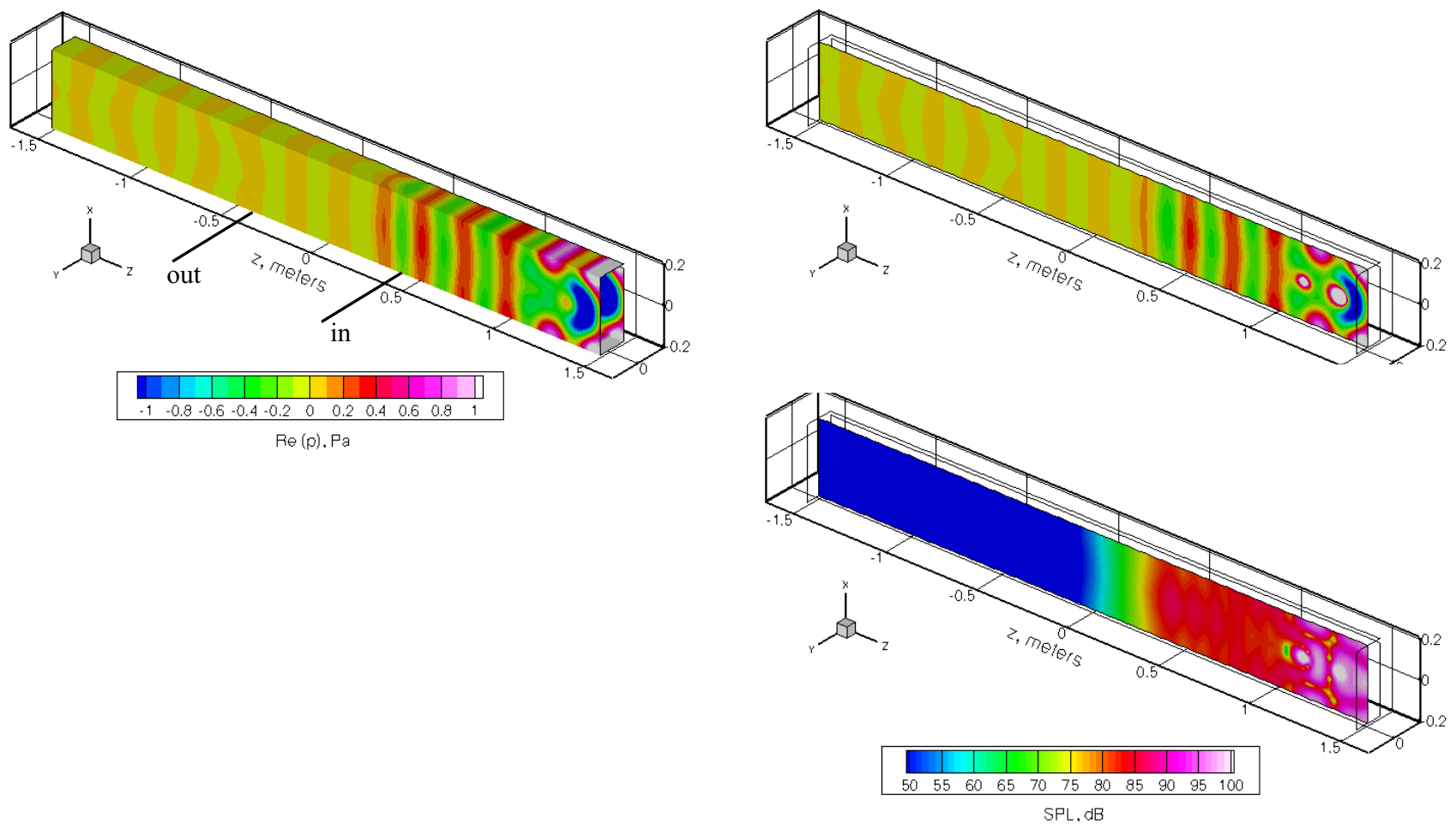

Figure 5. - Mode propagation within a straight duct with partially lined side walls, $Z=(0.228-0.137 i)(\rho c)$; $f=1500 \mathrm{~Hz}, M_{\infty}=0.0$, dominant $(0,0)$ mode. 

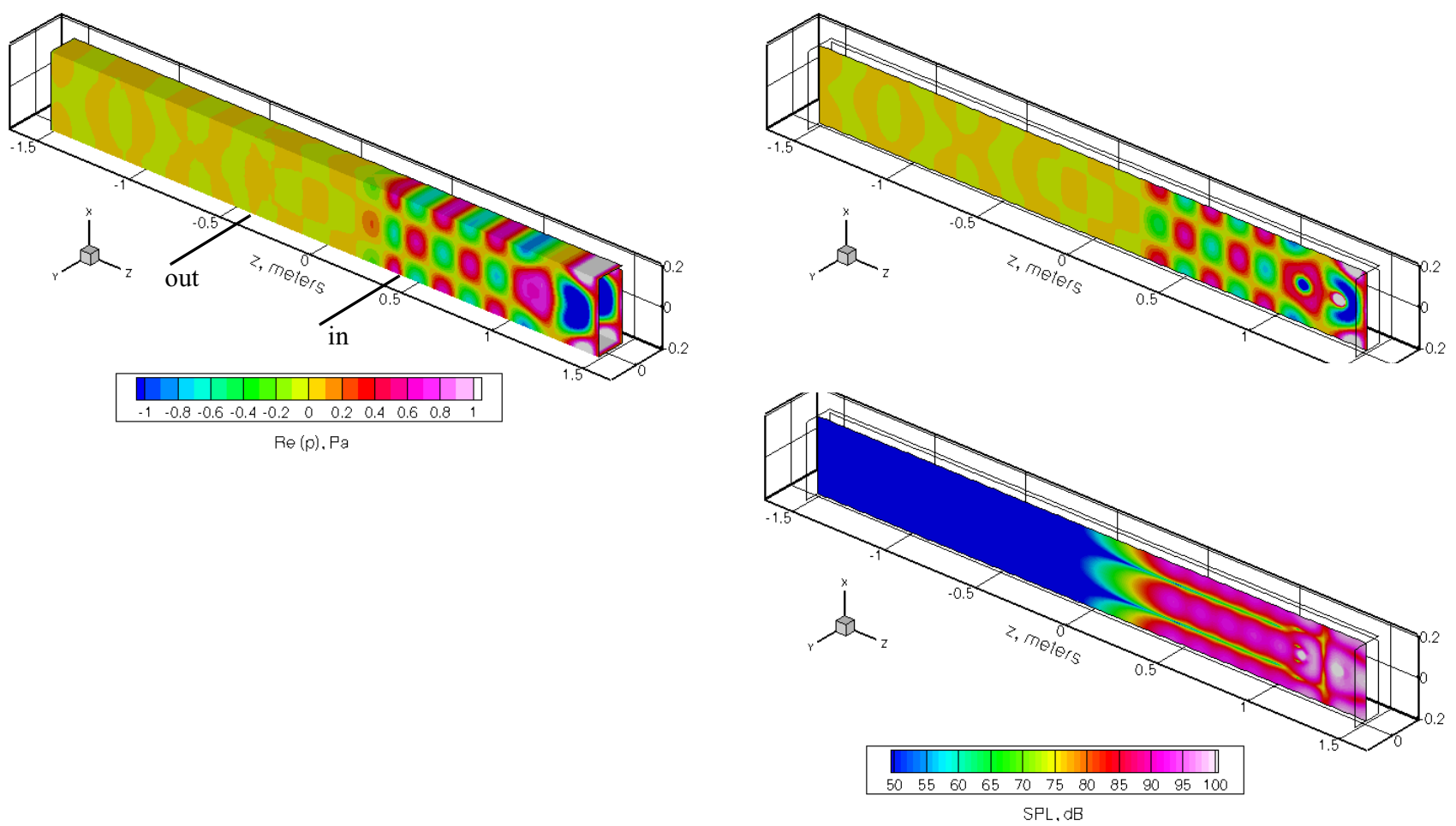

Figure 6. - Mode propagation within a straight duct with partially lined side walls, $Z=(0.228-0.137 i)(\rho c)$; $f=1500 \mathrm{~Hz}, M_{\infty}=0.0$, dominant $(2,0)$ mode.
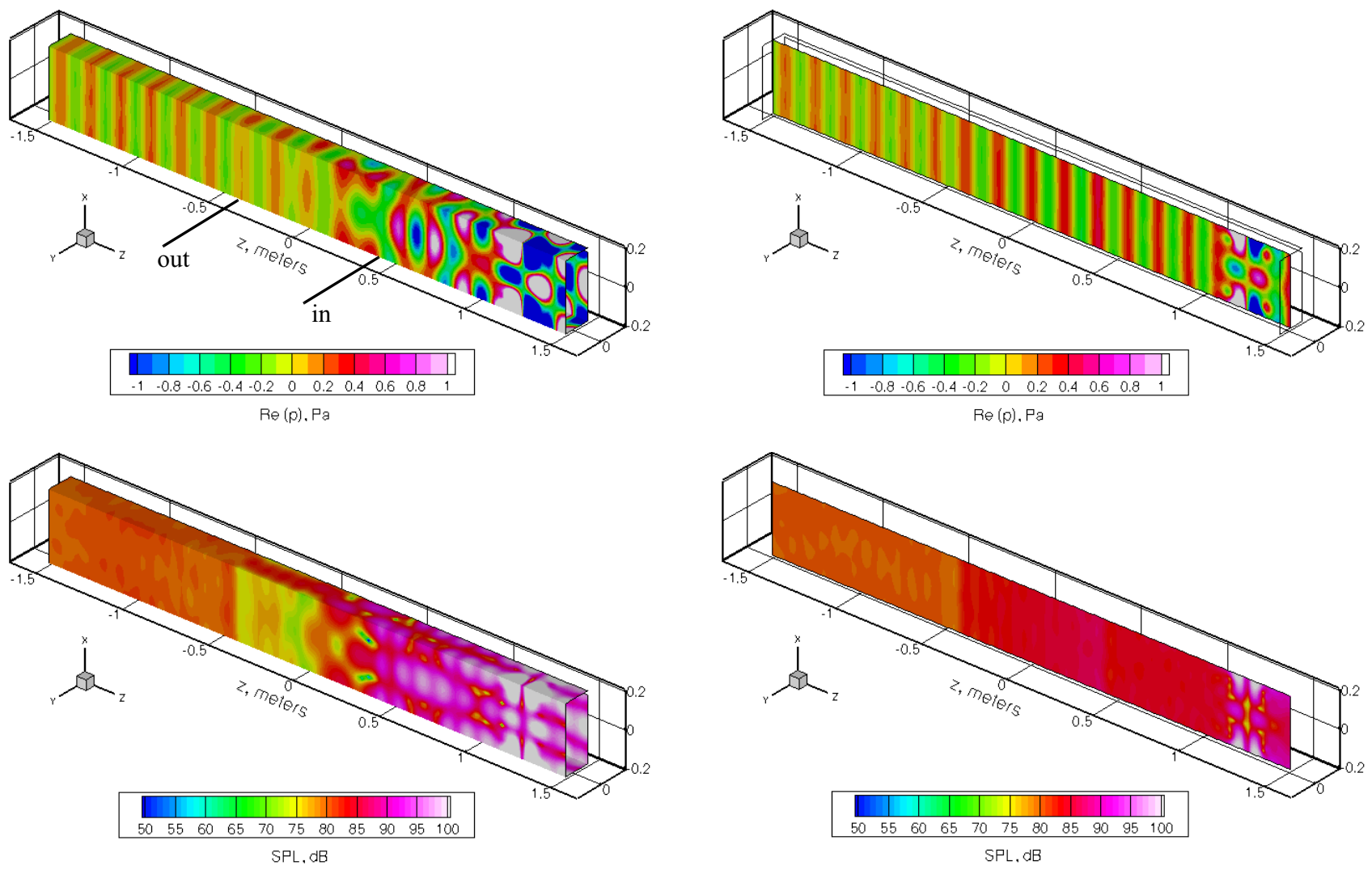

Figure 7. - Mode propagation within a straight duct with partially lined side walls, $Z=(0.115+0.347 i)(\rho c)$; $f=2000 \mathrm{~Hz}, M_{\infty}=0.0$, dominant $(2,1)$ mode. 
The effects of a uniform background flow on liner performance are presented in Figures 8 through 11. For a dominant plane wave at $f=1500 \mathrm{~Hz}$ (Figure 8), the introduction of flow appears to enhance the effectiveness of the liner in the region near the test section entrance. Most likely, this is a result of the changes in wavelength and/or pressure amplitude that occur in the presence of flow. The peak attenuation through the test section did not seem to be affected, remaining at about $30 \mathrm{~dB}$. No appreciable effects on liner behavior due to flow for a dominant $(2,0)$ mode at $f=1500$ $\mathrm{Hz}$ can be observed (Figure 9). The absence of noticeable changes may be caused by the imposition of a non-optimum liner impedance on the test section walls - the same value educed for the dominant plane wave was used for the $(2,0)$ mode as well, since the experimental data set did not contain information for this mode/frequency combination.

Flow effects on liner performance for a dominant $(2,1)$ mode at $f=2000 \mathrm{~Hz}$ are depicted in Figures 10 and 11. When the surface impedance does not include the effects of flow (Figure 10), the change in acoustic behavior inside the duct is similar to that for a dominant plane wave: the liner is slightly more effective near the test section inlet. When the effects of flow are included in the impedance (Figure 11), liner performance is noticeably improved, resulting in further attenuation of the downstream propagating plane wave. This effect is apparent in the acoustic pressure contours of Figure 11. Note also from the SPL contours that the attenuation through the test section increases to approximately $20 \mathrm{~dB}$. It has been determined ${ }^{7}$ that the presence of flow alters surface impedance by increasing resistance, $R$, and shifting reactance, $X$, to higher frequencies. The magnitude of the shift depends on the location of a particular excitation frequency on the $X(f)$ curve for the L01 liner: $1500 \mathrm{~Hz}$ may fall on a region of slowly varying reactance, that is, near the resonant frequency of the liner, whereas $2000 \mathrm{~Hz}$ may be within a region of rapidly changing reactance. The addition of flow also shifts peak attenuation to higher frequencies, which may result in a considerable reduction in plane wave amplitude, as seen in Figure 11.
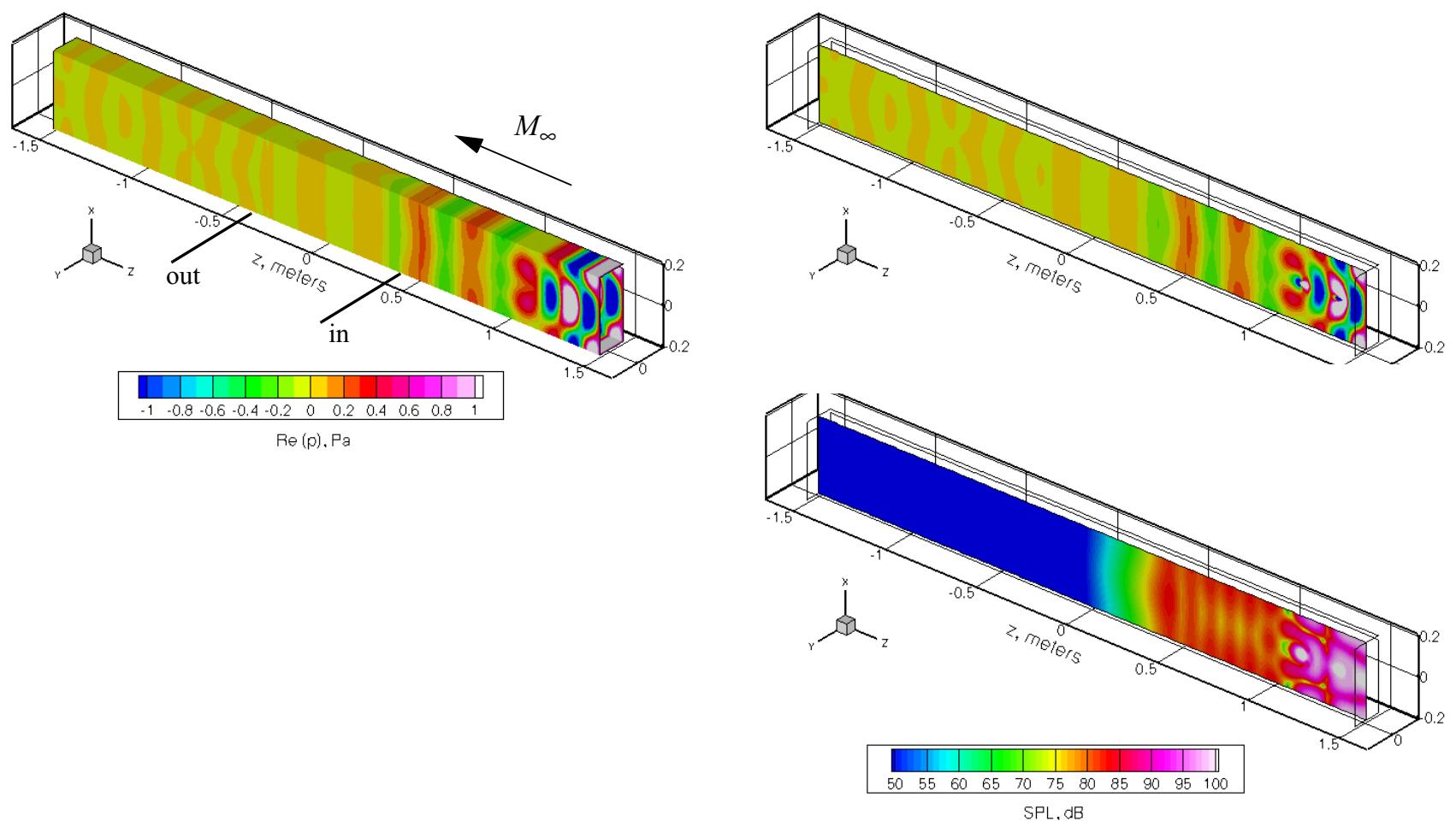

Figure 8. - Mode propagation within a straight duct with partially lined side walls, $Z=(0.761-0.427 i)(\rho c)$; $f=1500 \mathrm{~Hz}, M_{\infty}=0.275$ (air flows in the -z direction), dominant $(0,0)$ mode. 

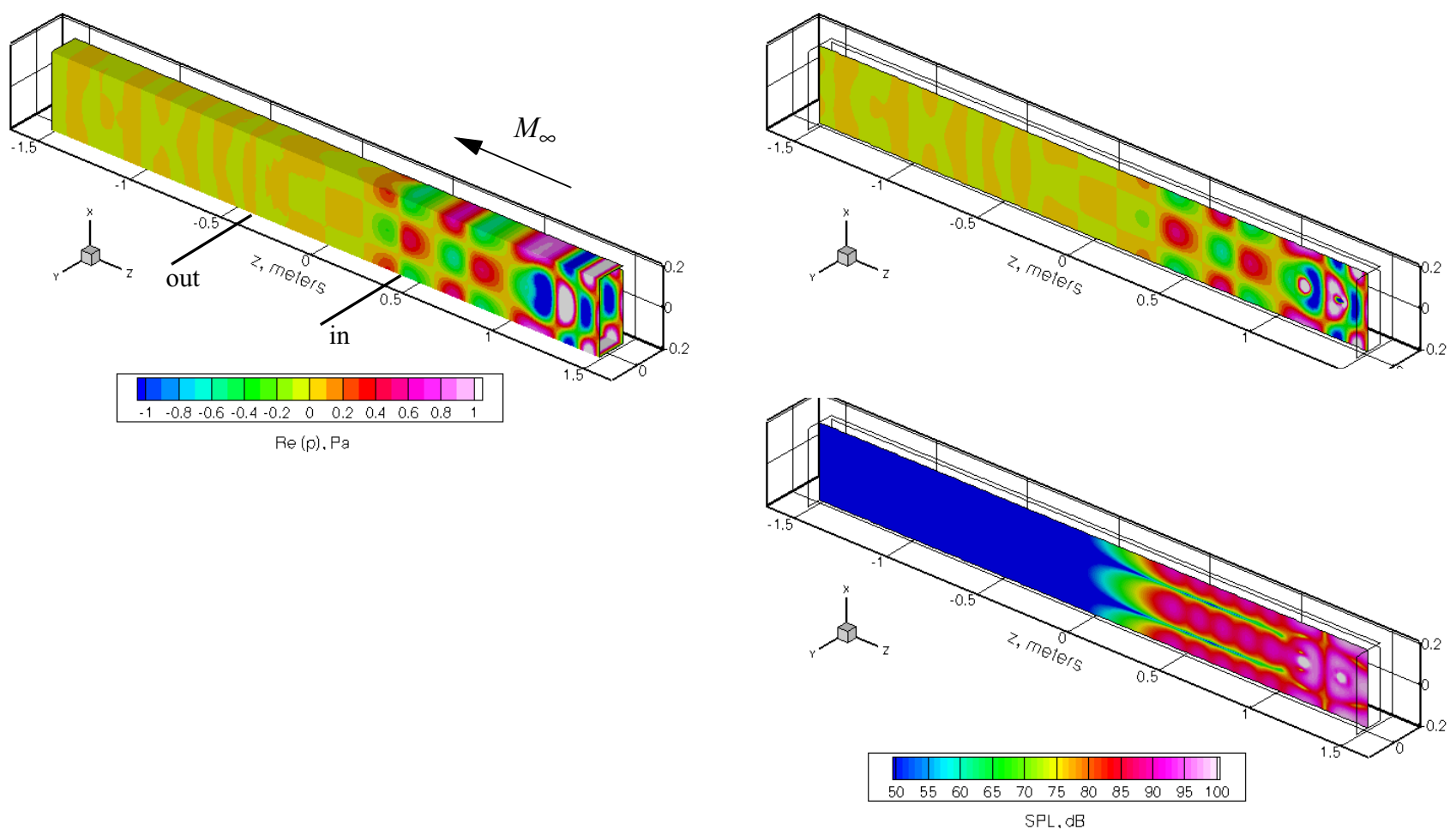

Figure 9. - Mode propagation within a straight duct with partially lined side walls, $Z=(0.761-0.427 i)(\rho c)$; $f=1500 \mathrm{~Hz}, M_{\infty}=0.275$ (air flows in the -z direction), dominant $(2,0)$ mode.
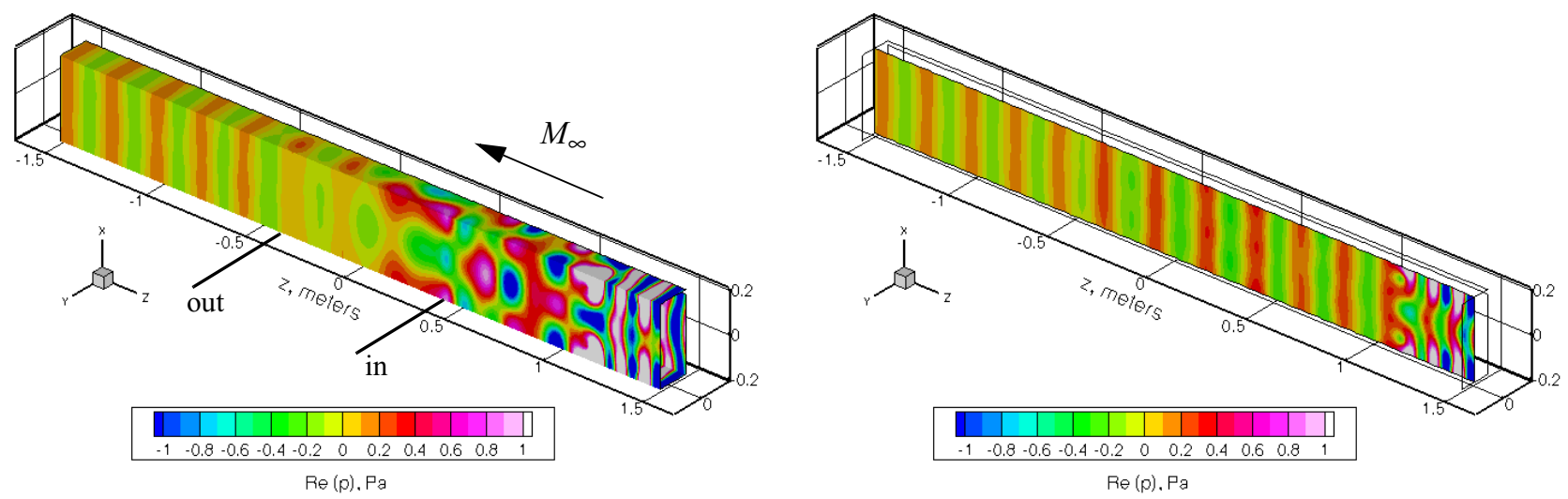

Figure 10. - Mode propagation within a straight duct with partially lined side walls, $Z=(0.115+0.347 i)(\rho c)$; $f=2000 \mathrm{~Hz}, M_{\infty}=0.275$ (air flows in the -z direction), dominant $(2,1)$ mode. 

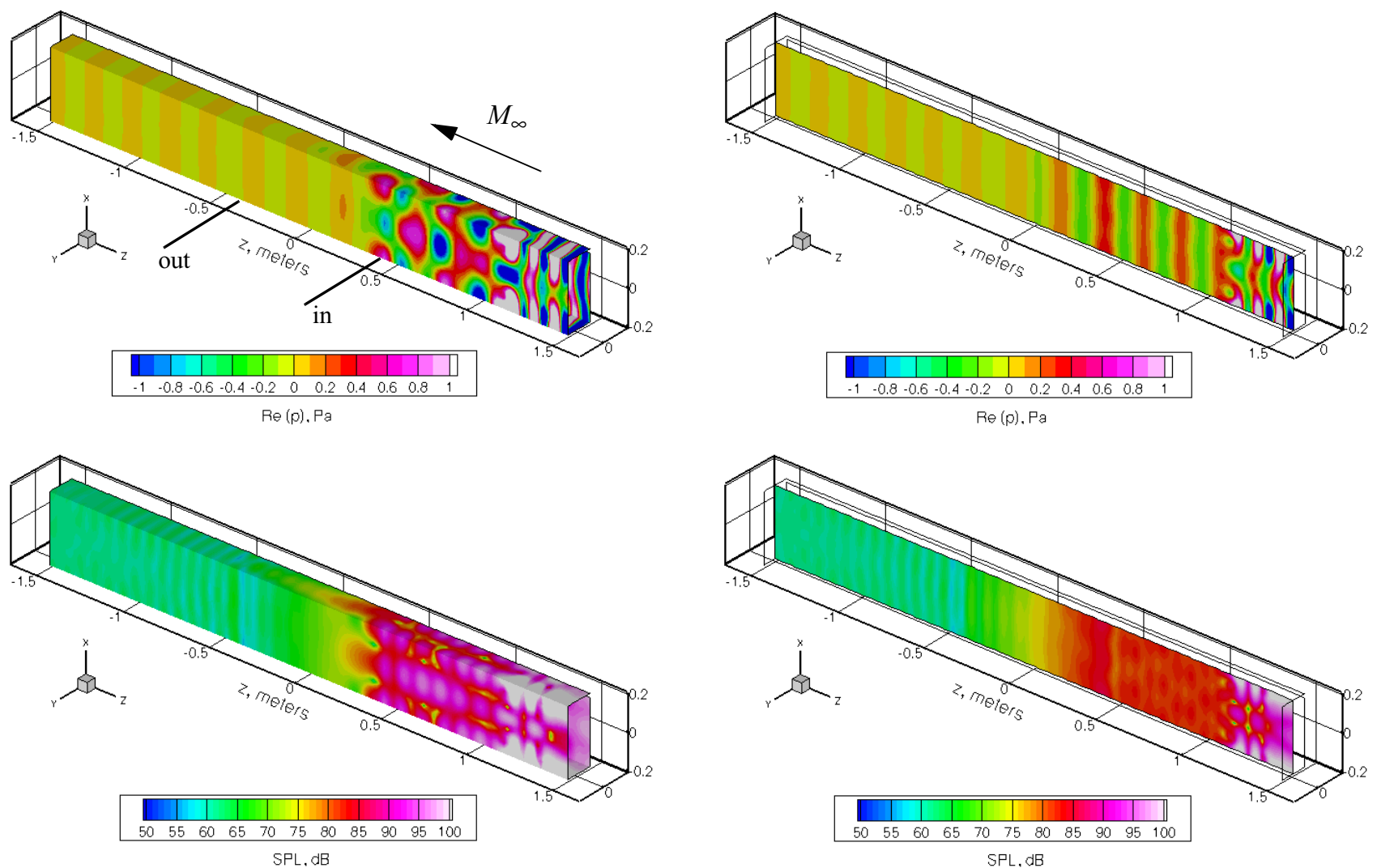

Figure 11. - Mode propagation within a straight duct with partially lined side walls, $Z=(0.730+0.130 i)(\rho c)$; $f=2000 \mathrm{~Hz}, M_{\infty}=0.275$ (air flows in the -z direction), dominant $(2,1)$ mode.

\section{Duct with Curved Test Section}

A primary goal of the CDTR project is the evaluation of duct curvature effects on noise propagation and liner performance. This is accomplished by modifying the test section so that the outlet plane is offset a distance of 0.0762 $m$ or $0.1524 m$, that is, $50 \%$ or $100 \%$ of the duct width, $L_{y}$ Simulations for a hardwall curved test section with an offset of $1.0 L_{y}$ are presented in Figure 12 for a dominant $(2,0)$ mode at an excitation frequency of $1500 \mathrm{~Hz}, M_{\infty}=0.0$. Note from the figure that interactions with the curved surfaces cause a slight decrease in wave amplitude aft of the test section. No appreciable change in liner behavior as a result of duct curvature can be observed (Figure 13).
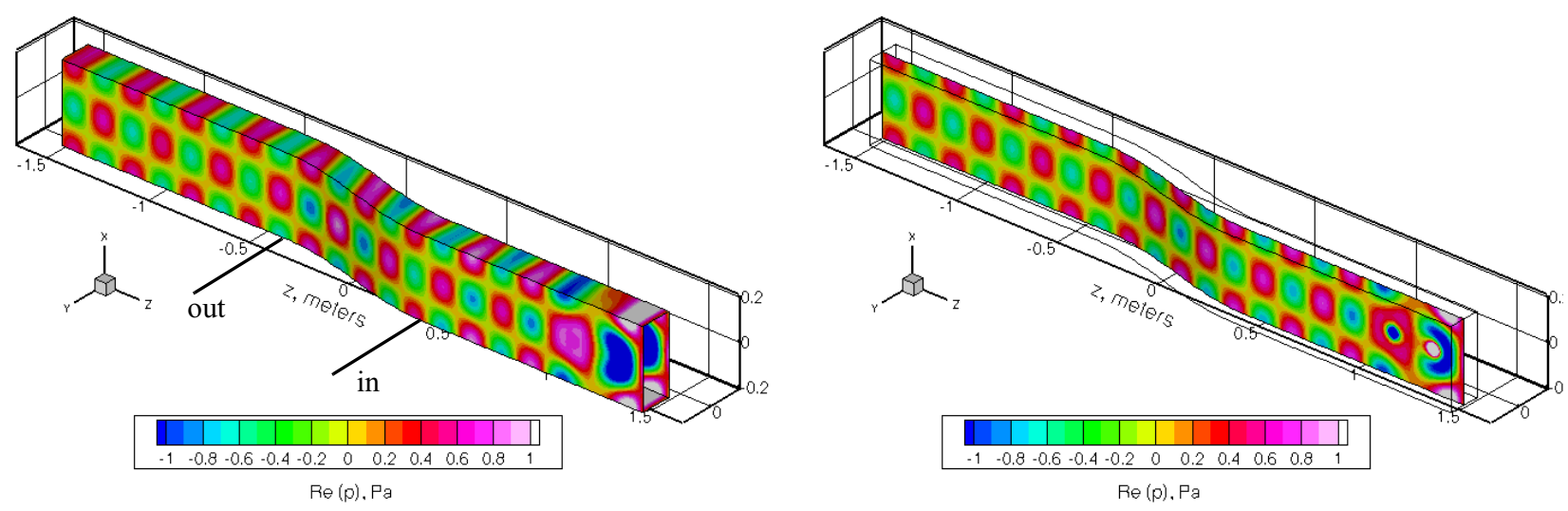

Figure 12. - Mode propagation within the CDTR test section with hard walls, duct offset $=1.0$ $L_{y} ; f=1500 \mathrm{~Hz}, M_{\infty}=0.0$, dominant $(2,0)$ mode. 

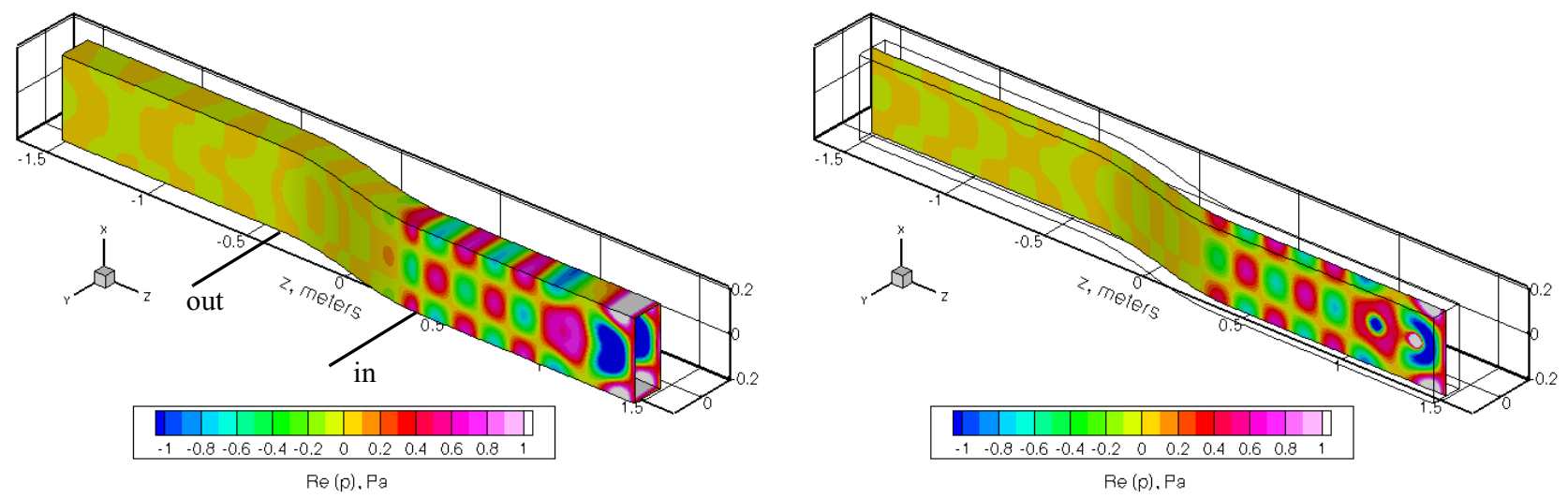

Figure 13. - Mode propagation within the CDTR test section with lined walls, $Z=(0.228-0.137 i)(\rho c)$, duct offset $=1.0 L_{y} ; f=1500 \mathrm{~Hz}, M_{\infty}=0.0$, dominant $(2,0)$ mode.

The effects of a more aggressively curved test section on mode propagation are depicted in Figures 14 and 15 for $f=1500 \mathrm{~Hz}, M_{\infty}=0.0$. The curvature follows a full sine wave of amplitude $0.5 L_{y}$. Note from Fig. 14 that the characteristics of a dominant plane wave propagating inside a hardwall duct are considerably altered inside the test section: secondary modes become apparent, causing an increase in wavelength at the walls and a decrease at the center. A dominant plane wave of reduced amplitude is re-established aft of the test section. Curvature causes a slight enhancement of the weaker modes inside the test section when a dominant $(2,0)$ mode is generated, as seen in Figure 15.
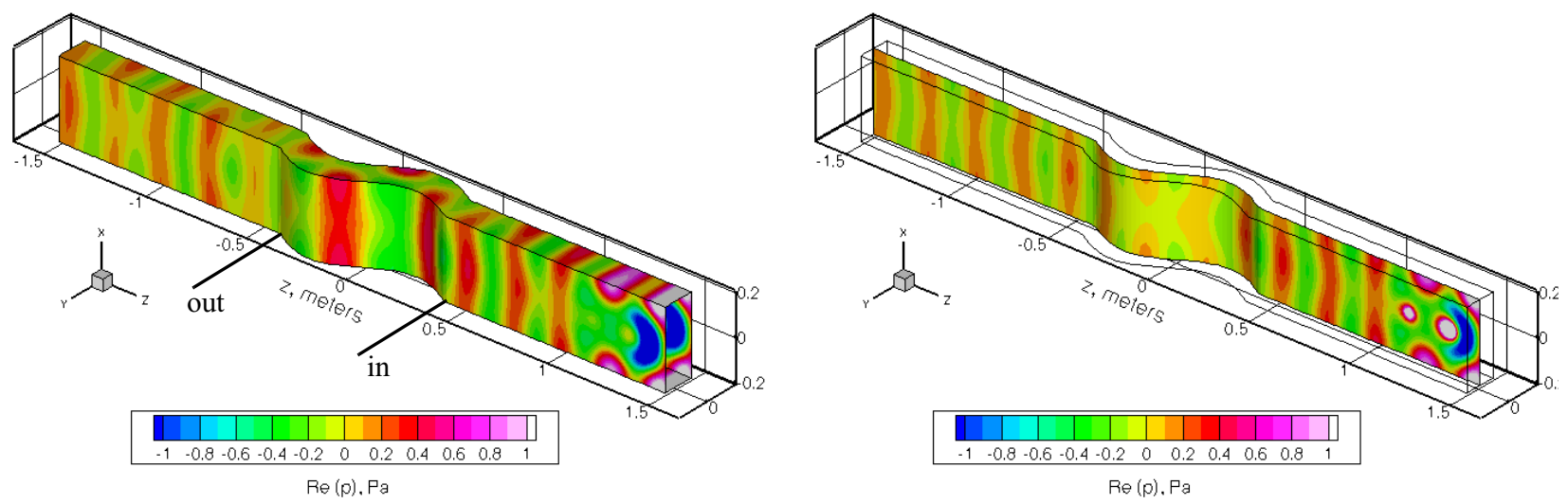

Figure 14. - Mode propagation within a sinusoidal test section with hard walls; $f=1500 \mathrm{~Hz}$, $M_{\infty}=0.0$, dominant $(0,0)$ mode.
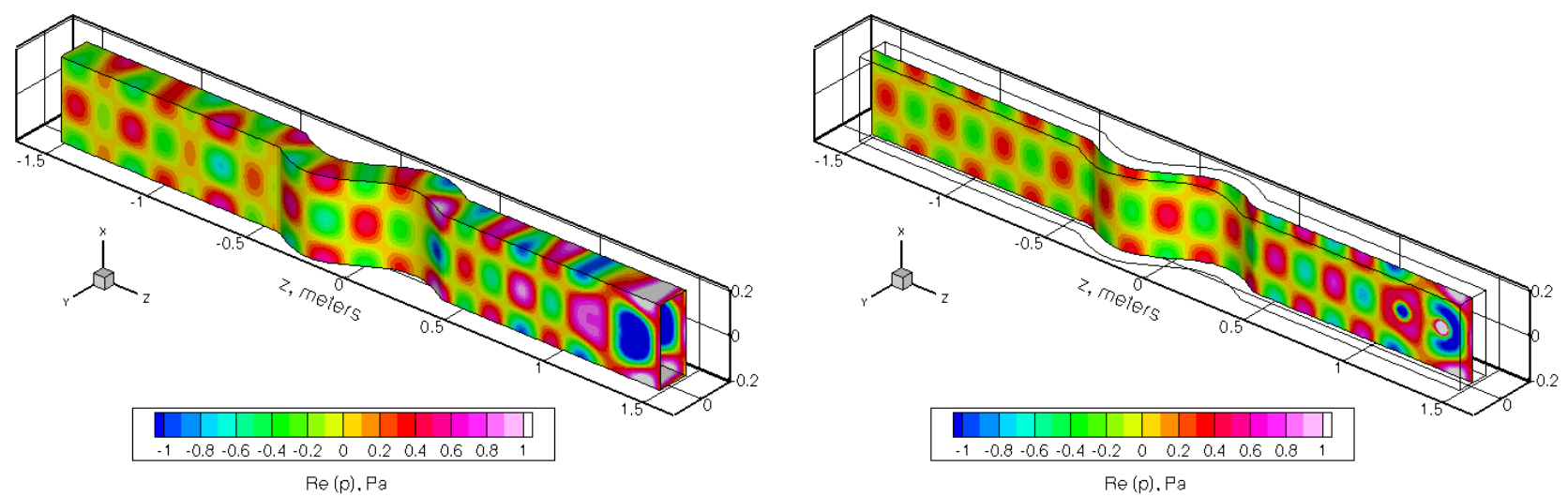

Figure 15. - Mode propagation within a sinusoidal test section with hard walls; $f=1500 \mathrm{~Hz}$, $M_{\infty}=0.0$, dominant $(2,0)$ mode. 
The characteristics of a wave traveling down the axis of a hardwall duct, as influenced by test section curvature, are depicted in Figure 16 for a dominant $(2,0)$ mode, $f=1500 \mathrm{~Hz}, M_{\infty}=0.0$. At this location within the duct, the salient features are a reduction in wave amplitude with increasing curvature, accompanied by changes in wavelength. Thus, it appears that duct curvature attenuates modal noise propagating within a waveguide of rectangular cross-section. Liner behavior does not seem to be affected by increased curvature of the test section (see Figures 17 and 18).

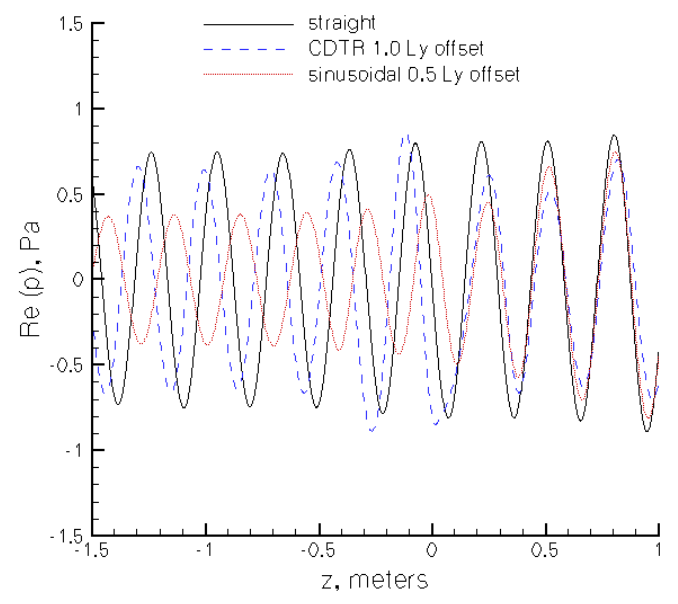

Figure 16. - Effect of test section curvature on a dominant $(2,0)$ mode, hardwall duct; $f=1500 \mathrm{~Hz}, M_{\infty}=0.0$.
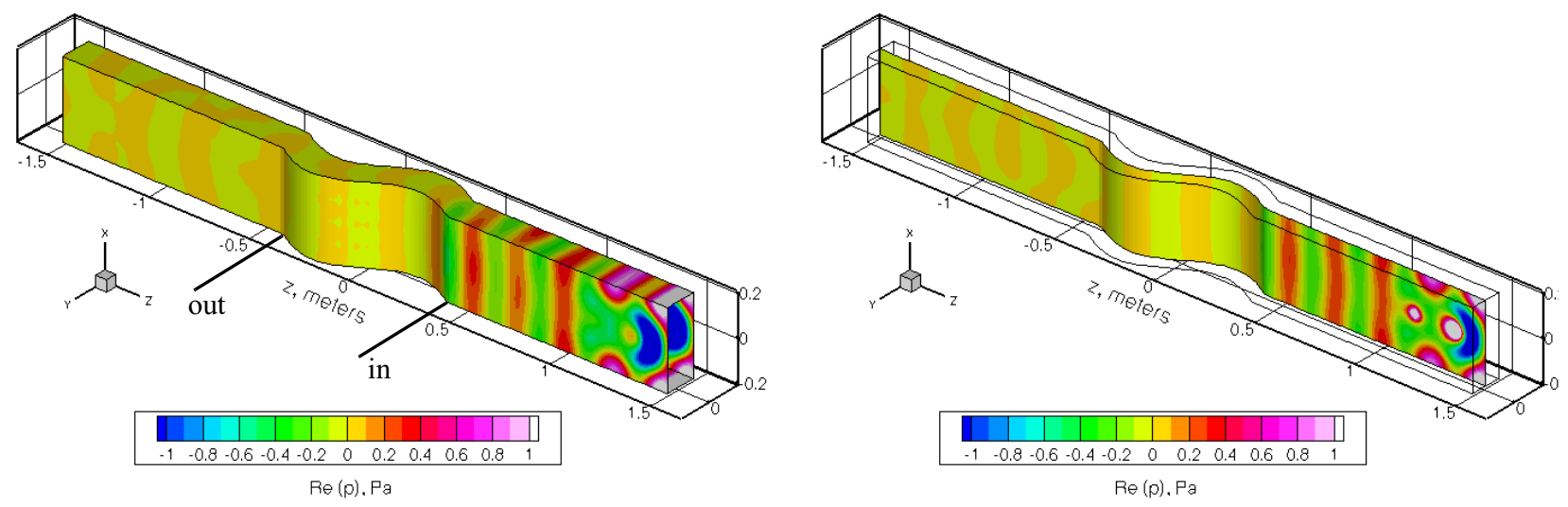

Figure 17. - Mode propagation within a sinusoidal test section with lined side walls, $Z=(0.228-0.137 i)(\rho c) ; f=1500 \mathrm{~Hz}, M_{\infty}=0.0$, dominant $(0,0)$ mode.
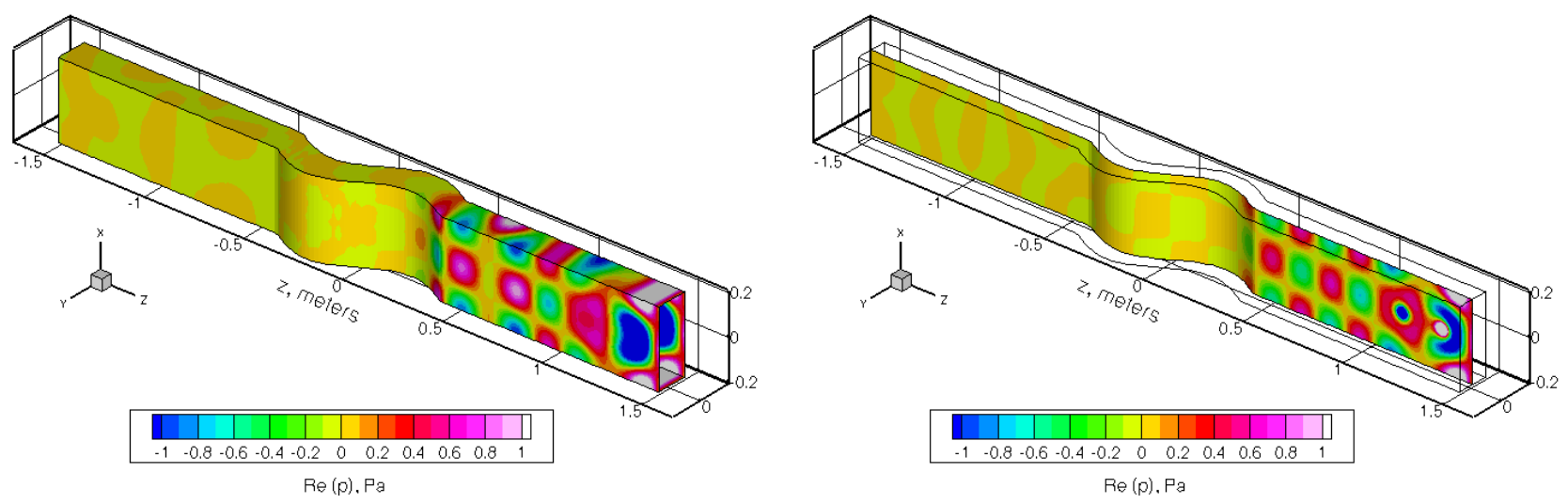

Figure 18. - Mode propagation within a sinusoidal test section with lined side walls, $Z=(0.228-0.137 i)(\rho \mathrm{c}) ; f=1500 \mathrm{~Hz}, M_{\infty}=0.0$, dominant $(2,0)$ mode. 


\section{Validation with Experimental Results}

The exterior Helmholtz BVP, which is solved using a collection of point multipoles located inside the scattering body (equivalent sources), can be transformed into an interior Helmholtz BVP by placing the equivalent sources outside the scattering surface. Doing so can substantially reduce the size of the BVP, since only the inner surfaces of the duct need to be considered. A Dirichlet boundary condition for initialization of acoustic pressure at the duct openings has been implemented into the FSC.

For this investigation, the incident sound field was input in the form of total acoustic pressure at the inlet $(z=$ $0.4064 \mathrm{~m})$ and outlet $(z=-0.4064 \mathrm{~m})$ planes of the test section. The pressures at these locations were synthesized from experimental measurements obtained with the CDTR for a target amplitude of $60 \mathrm{~Pa}(130 \mathrm{~dB})$ and a mode amplitude ratio (MAR) $)^{\ddagger}>10 \mathrm{~dB}$. The size of the input planes, $12 \times 30$, is sufficient for proper data resolution up to $2400 \mathrm{~Hz}$ (current frequency limit for the rig).

The results presented here correspond to a test section with hard walls, without flow. Although experimental data were available for lined side walls, the impedances educed with the GIT are incompatible with the formulation of the interior BVP used for this study. A proper comparison between simulated and experimental results for ducts with soft walls can be performed only if the impedances are educed with the FSC using the given synthesized pressures at the duct openings. Such an endeavor is beyond the scope of this paper.

Simulated acoustic pressures at the walls and bisecting plane of an unlined straight test section for a dominant ( 0 , 0 ) mode at $f=1500 \mathrm{~Hz}$, in the absence of flow, are depicted in Figure 19. Note that the code is able to calculate the proper modal behavior inside the duct using the Dirichlet boundary condition. Comparisons between calculated and measured acoustic pressures at the duct openings are presented in Figure 20. The agreement is excellent.

Simulations for a dominant $(2,0)$ mode at $1500 \mathrm{~Hz}$, without flow, are presented in Figures 21 and 22. Note from the pressures at the surfaces and bisecting plane (Figure 21) that the correct mode propagates through the test section. Agreement between calculated and experimental pressures at the duct openings (Figure 22) is excellent.
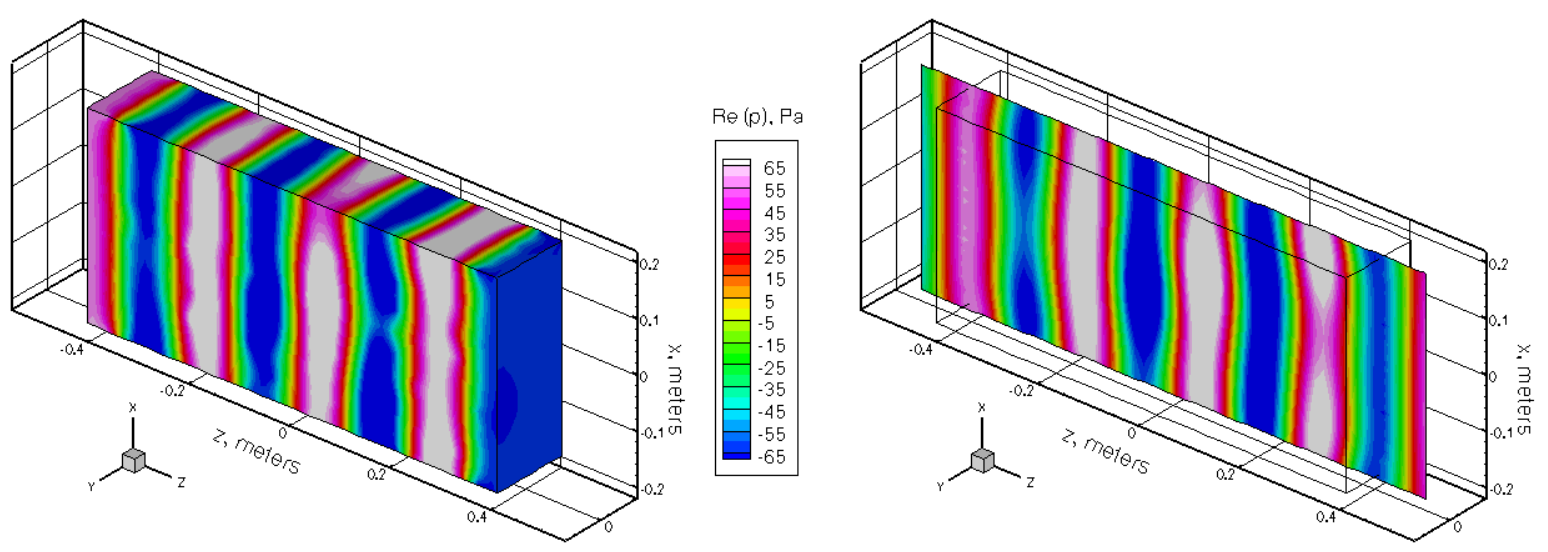

Figure 19. - Wave propagation within straight CDTR test section with hard walls; $f=1500 \mathrm{~Hz}$, $M_{\infty}=0.0$, dominant $(0,0)$ mode.

$\$$ MAR is the ratio of the target mode's amplitude to the amplitude of the next strongest mode, expressed in $\mathrm{dB}$. The target mode is considered dominant if MAR $>10 \mathrm{~dB}$. 


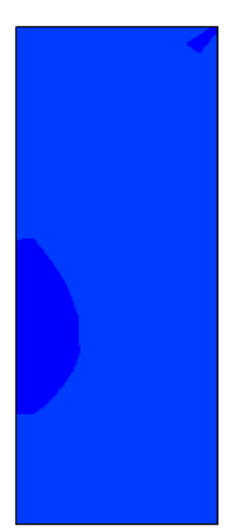

FSC

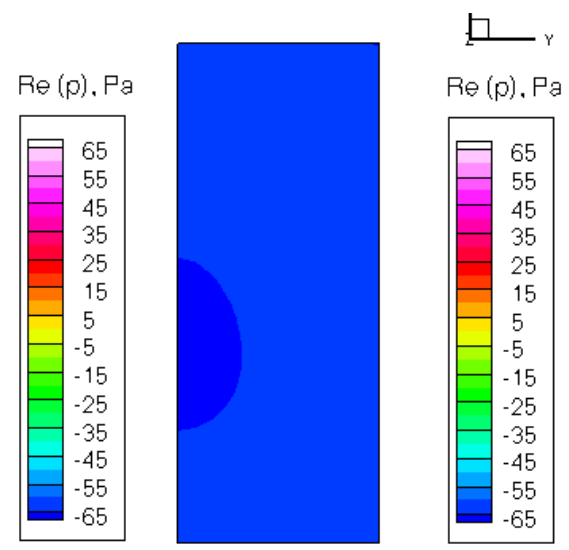

Experimental

(a) Inlet plane

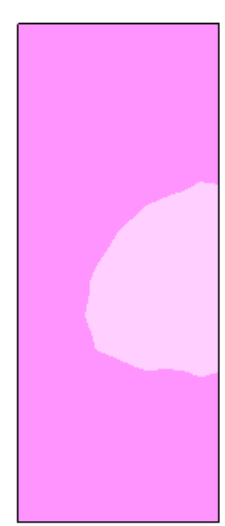

FSC

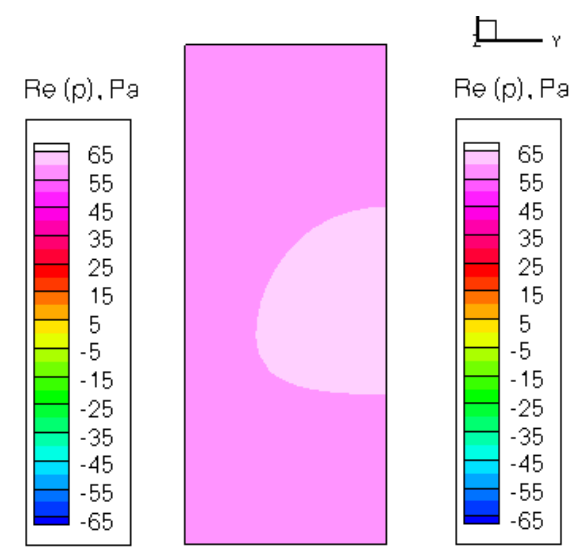

Experimental

(b) Outlet plane

Figure 20. - Comparison of total acoustic pressure at the test section openings, hard walls; $f=1500 \mathrm{~Hz}, M_{\infty}=0.0$, dominant $(0,0)$ mode.
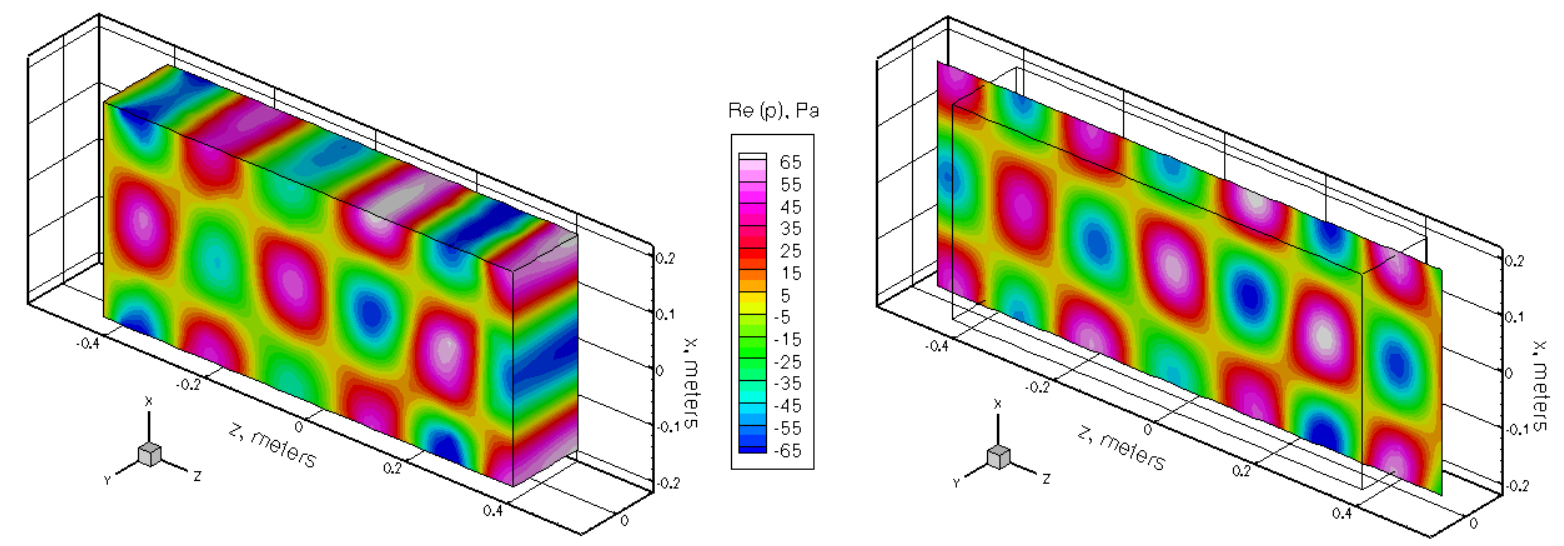

Figure 21. - Wave propagation within straight CDTR test section with hard walls; $f=1500 \mathrm{~Hz}$, $M_{\infty}=0.0$, dominant $(2,0)$ mode.

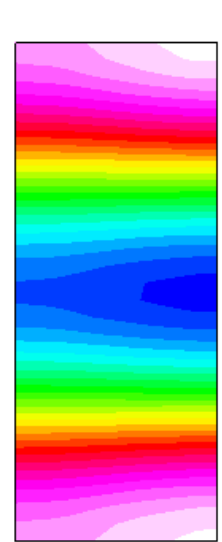

FSC

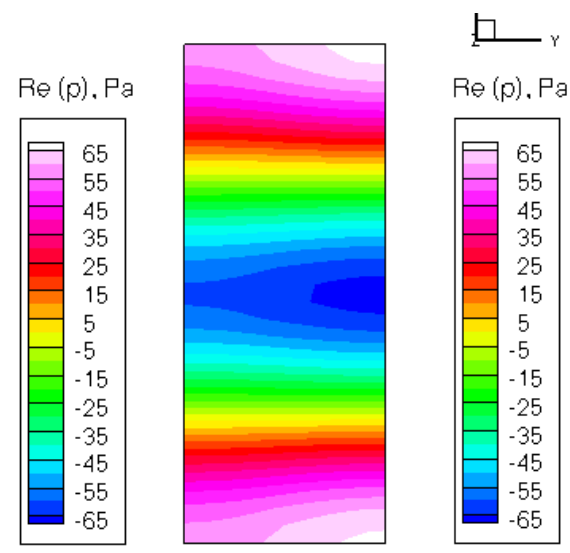

Experimental

(a) Inlet plane

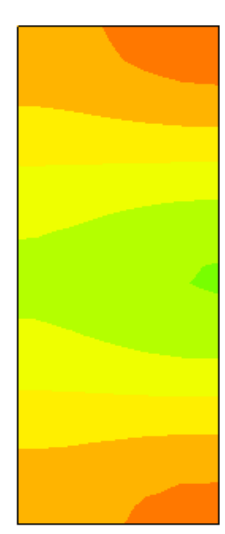

FSC

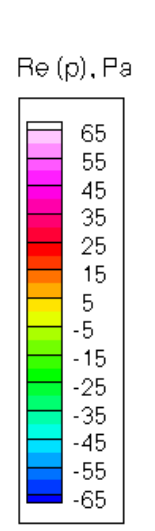

(b) Outlet plane

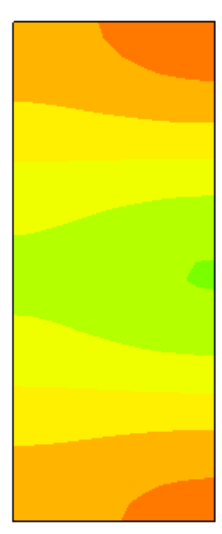

Experimental

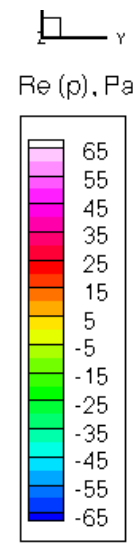

Figure 22. - Comparison of total acoustic pressure at the test section openings, hard walls; $f=1500 \mathrm{~Hz}, M_{\infty}=0.0$, dominant $(2,0)$ mode. 


\section{Concluding Remarks}

A study aimed at validating the Fast Scattering Code as a duct noise predictor has been undertaken. The effects of duct curvature, acoustic treatment, and uniform background flow have been included. Hardwall duct modes were used as the incident acoustic field for exterior BVP calculations. Comparisons between analytical and simulated results for a selected set of modes and frequencies indicate that the FSC is capable of generating the correct modal behavior inside the duct. Liner effects were incorporated through the use of a sound absorbing boundary condition at the scattering surfaces. The main effects of liner technology - changes in modal content and a reduction in acoustic pressure amplitude - are apparent in the simulations. The addition of curvature to the test section resulted in a localized amplification of weaker modes, and a reduction in amplitude for aft propagating waves. A Dirichlet boundary

condition for use with interior BVPs has been added to the code. Comparisons between simulated and experimental results for a straight, hardwall test section without flow are excellent.

\section{Acknowledgments}

Development of the Fast Scattering Code is being sponsored by the Aeroacoustics Branch of the NASA LaRC. The authors wish to thank Michael G. Jones, of the Structural Acoustics Branch, and Carl H. Gerhold, of the Aeroacoustics Branch, for their valuable input on duct acoustics and experimental measurements with the GIT and CDTR.

\section{References}

1. Dunn, M. H., and Tinetti, A. F., “Aeroacoustic Scattering Via the Equivalent Source Method,” AIAA 2004-2937, May 2004.

2. Tinetti, A. F., and Dunn, M. H., “Aeroacoustic Noise Prediction Using the Fast Scattering Code," AIAA 20053061, May 2005.

3. Tinetti, A. F., Dunn, M. H., and Pope, D. S., Fast Scattering Code (FSC) User's Manual, Version 2.0, NASA CR2006-214510, October 2006.

4. Gerhold, C., Cabell, R., and Brown, M., "Development of an Experimental Rig for Investigation of Higher Order Modes in Ducts," AIAA-2006-2637, May 2006.

5. Morse, P. M., and Ingard, K. U., Theoretical Acoustics, McGraw-Hill Book Company, 1968.

6. Jones, M. G., Parrott, T. L., and Watson, W. R., "Comparison of Acoustic Impedance Eduction Techniques for Locally-Reacting Liners," AIAA 2003-3306, May 2003.

7. Gerhold, C., Brown, M., Watson, W., and Jones, M., "Investigation of Liner Characteristics in the NASA Langley Curved Duct Test Rig,” AIAA 2007-3532, May 2007.

8. Dowling, A. P., and Ffowcs-Williams, J. E., Sound and Sources of Sound, Ellis Horwood Limited, John Wiley and Sons, 1983.

9. Mangiarotty, R. A., "Acoustic Lining Concepts and Materials for Engine Ducts," Journal of the Acoustical Society of America, Vol. 48, No. 3, pp.780-794, 1970.

10. Atvars, J., and Mangiarotty, R. A., Parametric Studies of the Acoustic Behavior of Duct-Lining Materials," Journal of the Acoustical Society of America, Vol. 48, No. 3, pp.815-825, 1970. 